\title{
Adaptive Control of a Hybrid Powertrain with Map-based ECMS
}

\author{
Martin Sivertsson*, Christofer Sundström*, and \\ Lars Eriksson* \\ * Vehicular Systems, Dept. of Electrical Engineering, Linköping \\ University, SE-58183 Linköping, Sweden, \{marsi,csu,larer\}@isy.liu.se.
}

\begin{abstract}
To fully utilize the fuel reduction potential of a hybrid powertrain requires a careful design of the energy management control algorithms. Here a controller is created using mapbased equivalent consumption minimization strategy and implemented to function without any knowledge of the future driving mission. The optimal torque distribution is calculated offline and stored in tables. Despite only considering stationary operating conditions and average battery parameters, the result is close to that of deterministic dynamic programming. Effects of making the discretization of the tables sparser are also studied and found to have only minor effects on the fuel consumption. The controller optimizes the torque distribution for the current gear as well as assists the driver by recommending the gear that would give the lowest consumption. Two ways of adapting the control according to the battery state of charge are proposed and investigated. One of the adaptive strategies is experimentally evaluated and found to ensure charge sustenance despite poor initial values.
\end{abstract}

Keywords: Hybrid Vehicles, Adaptive Control, Automotive Control, Optimal Control

\section{INTRODUCTION}

A hybrid powertrain utilizes at least two separate energy converters. This has the potential to significantly increase the efficiency of the powertrain. The key to utilizing the full potential of the powertrain lies in the design of the control algorithm. The goal in hybrid powertrain control is normally to minimize the fuel consumption while maintaining the battery State of Charge (SOC) within prescribed limits, sometimes with addition of constraints regarding emissions.

This paper develops an adaptive Equivalent Consumption Minimization Strategy (ECMS), based on Musardo and Rizzoni (2005), and applies it to the Haldex electric Torque Vectoring Drive (eTVD). The optimal torque distribution is calculated offline and stored in tables and the effects of discretization on the fuel consumption is studied. Then two ways of adapting the control to maintain the SOC within the desired limits are investigated.

\subsection{The Haldex eTVD and the test vehicle}

The system used for modeling, simulation, and experimental evaluation is a SAAB 9-3 XWD with a 2.0L turbo charged spark ignited combustion engine and a six-speed manual gear-box (GB), fitted with the eTVD.

The eTVD is a system designed to combine all-wheel drive (AWD) with hybrid functionality. It also has the ability to control the torque distribution on the rear wheels individually, which is useful to prevent underand over-steering. In the eTVD concept the combustion engine (ICE) and main electric motor (EM) are connected electrically to each other via the generator (ISG) and

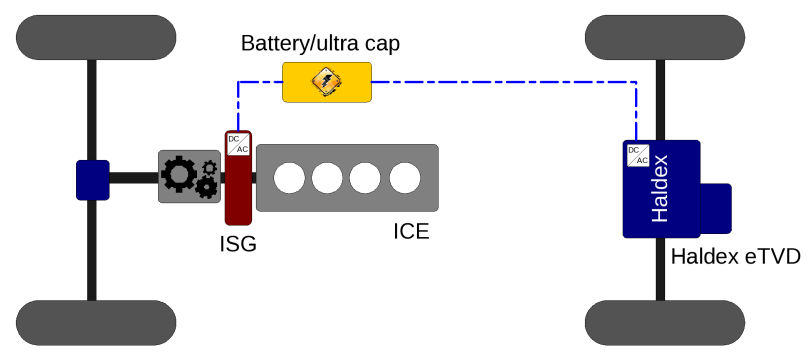

Fig. 1. The architecture of the Haldex eTVD concept. The EM is labeled Haldex in the figure. There are two energy paths between the ICE and EM.

mechanically via the wheels, see Fig. 1. The architecture of the system thus resembles that of a split hybrid but since the components in the test vehicle are dimensioned for AWD the series hybrid functionality of the vehicle is reduced. A more fitting description would be advanced parallel hybrid since the powertrain can be viewed as a parallel hybrid with an extra degree of freedom in choosing which energy converter to use during load shifting since the ISG is added to the powertrain.

\section{VEHICLE MODEL}

For the purpose of investigating different control strategies and their fuel consumptions, a quasistatic model approach is used. This is partly due to simplicity but also because it is well suited for both DDP and ECMS. In a backward facing quasistatic approach the speed is known from the driving cycle. When the speed is known the torque required at the wheels to follow the driving cycle is calculated through a longitudinal vehicle model: 


$$
T_{r e q}=r_{w}(\underbrace{\frac{\rho_{a}}{2} C_{D} A_{f} V(t)^{2}}_{F_{a i r}}+\underbrace{m g f_{r}}_{F_{\text {roll }}}+\underbrace{m \dot{V}(t)}_{F_{\text {acc }}}+\underbrace{\dot{V}(t) \frac{J_{w}}{r_{w}^{2}}}_{F_{w i}})
$$

where $F_{\text {air }}$ is the aerodynamic drag, $F_{\text {roll }}$ the rolling resistance, $F_{w i}$ the inertia of the wheels, and $F_{a c c}$ is the acceleration force. Force from road grade is neglected.

\subsection{Components}

The control signals of the system are the energy converter torques $T_{I C E}, T_{I S G}, T_{E M}$, and gear $\gamma_{G B}$. The components (ICE, EM, ISG and GB) are all modeled with a power balance and efficiency, $P_{\text {out }}=P_{i n} \eta$, where the efficiencies, $\eta$, are assumed to be known and account for all losses in the component. The efficiency $\eta_{G B}$ is assumed constant while the efficiencies of the energy converters are shown in Fig. 2. The battery is modeled as a Thevenin equivalent circuit with open circuit voltage $U_{o c}(S O C)$, coloumbic charge efficiency $\eta_{c}(S O C)$, and constant internal resistance $R_{i}$. The battery in the test vehicle outputs its SOC, thus the SOC is assumed to be known. The power required by the auxiliary units, $P_{a u x}$, is assumed constant. For more details about the modeling see Sivertsson (2010).

\section{REFERENCE CONSUMPTIONS}

As a reference for the implemented optimization, deterministic dynamic programming (DDP) as described in Guzzella and Sciarretta (2007) is used. Time and SOC are discretized with a step length of $1 \mathrm{~s}$ and $0.02 \%$ respectively. The SOC discretization is chosen so that one step roughly equals the change in SOC from the auxiliary units during 1s. The operating points from the DDP solution to NEDC are shown in Fig. 2. Interesting to note is the efficient use of the ISG in load shift and that almost all the EMs operating points during braking are on, or close, to the torque limit. This is a result of the EM and ISG primarily being designed for torque vectoring and AWD and not fuel economy.

To evaluate the performance of the real-time control, the consumption as a strictly AWD vehicle is used. For that purpose a control is used where the gear that results in the lowest consumption at each time is engaged. The EM is assumed to be unused both in traction and braking, thus this mode corresponds to pure ICE propulsion.

\section{THE ECMS}

In ECMS, proposed in Paganelli et al. (2001) and Paganelli et al. (2002), the sum of fuel and fuel equivalent of the electrical power is minimized. Since fuel and battery power are not directly comparable an equivalence factor, $\lambda$, is used. The function to be minimized can be written as:

$$
H=P_{f}\left(T_{I C E}, \gamma_{G B}\right)+\lambda(t) P_{b a t t}\left(T_{E M}, T_{I S G}, \gamma_{G B}\right)
$$

Under the assumption that the battery efficiency is independent of SOC, the equivalence factor $\lambda$ remains approximately constant along the optimal trajectory. Therefore the optimization problem is reduced to finding the constant $\lambda$ that approximates the optimal trajectory of a given driving cycle. Since the characteristics of the battery depends on if the battery is charging or discharging, $\lambda$
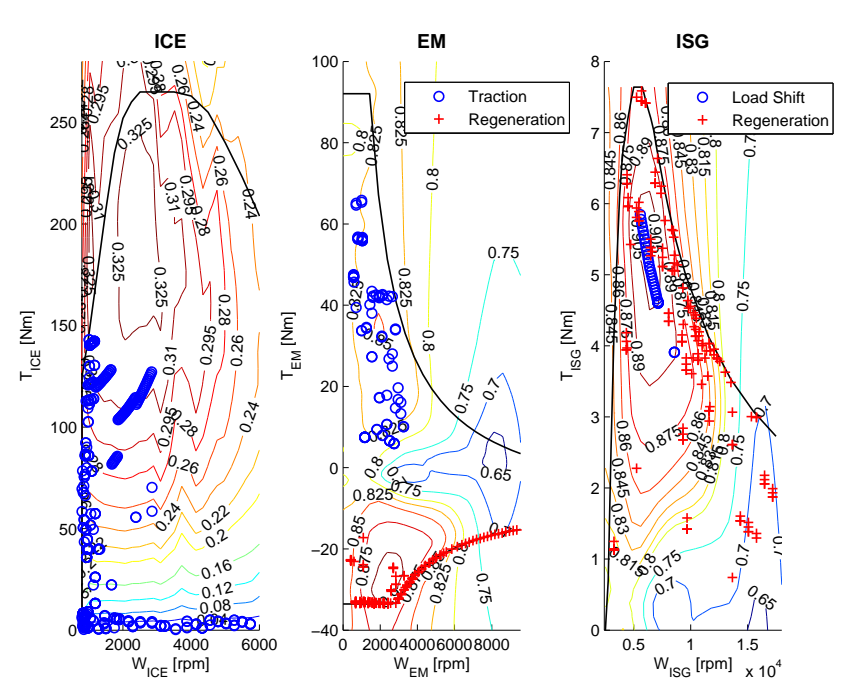

Fig. 2. Efficiencies of the ICE, EM, and ISG, as well as the operating points of the three energy converters on the NEDC from the DDP solution

is sometimes replaced by two constants. It is however shown in Musardo and Rizzoni (2005) that one constant suffices to get a good approximation on a given driving cycle, which is the approach selected here. For more details on ECMS see Guzzella and Sciarretta (2007); Pisu and Rizzoni (2007); Sciarretta et al. (2004).

As a consequence of the discussion above the strategy for selecting the control inputs becomes:

Subject to:

$$
\left[T_{I C E}, T_{I S G}, T_{E M}, \gamma_{G B}\right]=\operatorname{argmin}(H)
$$

$$
\begin{aligned}
& T_{r e q}=\eta_{G B} \gamma_{G B}\left(T_{I C E}-\gamma_{I S G} T_{I S G}\right)+\gamma_{E M} T_{E M} \\
& T_{E M, \min }\left(\omega_{E M}\right) \leq T_{E M} \leq T_{E M, \max }\left(\omega_{E M}\right) \\
& 0 \leq T_{I C E} \leq T_{I C E, \max }\left(\omega_{I C E}\right) \\
& 0 \leq T_{I S G} \leq T_{I S G, \max }\left(\omega_{I S G}\right) \\
& P_{\text {batt,min }}(S O C) \leq P_{\text {batt }} \leq P_{\text {batt,max }}(S O C)
\end{aligned}
$$

\subsection{Offline Optimization}

Instead of solving the computationally demanding three degree of freedom problem in (3)-(4) for all possible controls in real-time, the optimization is performed offline and the result is tabulated. In the real-time implementation the control system interpolates in the stored data to find the optimal torque distribution.

In the offline calculations the three parameters that the ECMS algorithm takes as input, i.e. vehicle speed, required torque, and equivalence factor, are discretized and the optimal torque distribution on the three energy converters, as well as the optimal gear, are calculated as a function of $\left(V, T_{r e q}, \lambda\right)$ for each point. Since, for each gear, it is a two degree of freedom problem, see (4a), it requires two tables for each gear, one for the ISG and one for the ICE. From these two tables the torque required from the EM can be calculated using (4a). With six gears, not including reverse, a total of 13 tables are calculated: six ICE, six ISG and one for the gear selection. So the system not only optimizes the torque distribution for the current gear, it also assists the driver by recommending the gear that would give the lowest fuel consumption. 


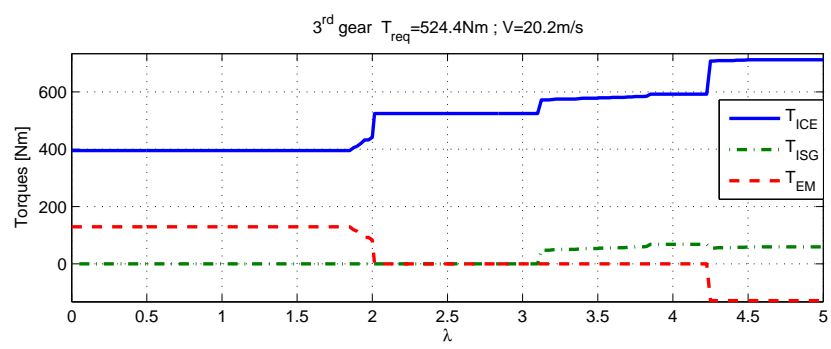

Fig. 3. The optimal torques on the energy converters as a function of $\lambda$ for a specific vehicle speed and required torque. All torques are calculated at the wheels.
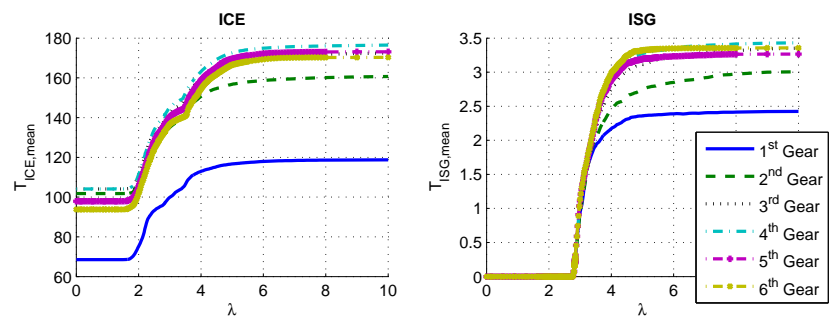

Fig. 4. The mean torque in each table changes as a function of $\lambda$.

\subsection{Table Discretization}

The real-time algorithm uses linear interpolation in the stored data and the assumption is thus that the solution is linear between two optima. As seen in Fig. 3 the solution is only piecewise linear between two $\lambda$ so the discretization has to be chosen with care. Fig. 4 shows how the mean torque in each table changes as a function of $\lambda$. Since it is desirable to reduce the memory requirements the idea is to make the $\lambda$ discretization sparser in the segments that could be considered linear and denser where it is clearly non-linear. From Fig. 4 it is clear that the ISG and ICE tables should have separate $\lambda$ discretizations since the ISG tables remain constant up until $\lambda \approx 3$ while the ICE tables only remain constant until $\lambda \approx 2$. To test how the loss of accuracy, introduced by reducing the size of the tables, affects the consumption, simulations are carried out on two sets of tables: one large set, $\mathcal{T}_{L}$, and one small set, $\mathcal{T}_{S}$. The $\mathcal{T}_{L}$ has the same $\mathrm{V}$ - and $T_{\text {req }}$-discretization as the offline optimization itself, but the $\lambda$-discretization is made sparser according to the strategy mentioned above. The $\mathcal{T}_{S}$-discretization is made sparser than $\mathcal{T}_{L}$ in all three directions. The memory requirement of $\mathcal{T}_{S}$ is roughly one eighth of that of $\mathcal{T}_{L}$.

\section{REAL-TIME IMPLEMENTATION DETAILS}

In the implementation the ECMS is only used in traction. During braking the main parameter that affects the ability to recuperate energy is the gear. However the time spent in braking is deemed too short to motivate a gear shift. Instead a heuristic brake control is used. Provided that the SOC is within limits the EM supplies the requested brake torque and if the EM is insufficient, the ISG, providing that a gear is engaged, also provides a regenerative torque and the rest is handled by the friction brakes.
Table 1. The decrease in fuel consumption with DDP compared to ECMS $S_{\text {opt }}$.

\begin{tabular}{|c|c|c|}
\hline & DDP: Free Gear & DDP: Fixed Gear \\
\hline NEDC & $4.0 \%$ & $0.2 \%$ \\
\hline FTP-75 & $10.1 \%$ & $2.3 \%$ \\
\hline
\end{tabular}

Since the test vehicle has a manual gearbox the gear selector recommends gears to the driver. In simulation the system follows the gear selector and shifts instantaneously without the use of a clutch. To avoid too frequent gear shifts (recommendation changes) a hysteresis is applied to the interpolated optimal gear.

\section{EQUIVALENCE FACTOR CONSIDERATIONS}

An equivalence factor $\lambda$ is considered optimal if the resulting $\triangle S O C=S O C\left(t_{\text {end }}\right)-S O C\left(t_{\text {start }}\right)=0$ within a specified tolerance. Since the speed in the driving cycles is discretized, the situation can arise where there exists no $\lambda$ that results in $\triangle S O C=0$. In those situations the method suggested in Chasse et al. (2009) is used, where the control switches between two $\lambda, \lambda_{1}$ leading to $\triangle S O C>0$ and $\lambda_{2}$ leading to $\triangle S O C<0$, at time $t$ to achieve $\triangle S O C=0$.

To evaluate the offline optimization a set of tables, $E C M S_{o p t}$, consisting of $\lambda_{o p t}$ for the NEDC and FTP-75 cycles is created. That is, no interpolation between two optima in the $\lambda$-direction is necessary. In Fig. 5 the SOC trajectories of ECMS $S_{\text {opt }}$ as well as DDP on the FTP-75 cycle is shown. Since the gear shifting of the DDP solution is highly unrealistic, a DDP Fixed Gear solution using the gear trajectory of $E C M S_{\text {opt }}$ is also shown. The $E C M S_{o p t}$ SOC trajectory still differs from the two DDP solutions. This is due to the offline optimization using only stationary operating points and average battery parameters. However, introducing SOC-dependency in the offline optimization would mean adding an extra dimension in each table and the decrease in consumption using DDP compared to $E C M S_{\text {opt }}$ is small, especially if a more realistic gear shifting strategy is used, as shown in Table 1.

Results from simulations on the NEDC and FTP-75 driving cycles are shown in Table 2. The increase in consumption for $\mathcal{T}_{S}$ and $\mathcal{T}_{L}$ compared to $E C M S_{\text {opt }}$, as well as the difference in $\lambda_{\text {opt }}$ values, confirm that the solution is not a linear function of $\lambda$. However, the increase in consumption is small, only $0.1 \%$ from making the $\lambda$-dimension sparser and $0.2-0.4 \%$ when the tables are made sparser in all three dimensions.

Even if the simulations show that the implemented ECMS produce a good result on a given driving cycle, close to that of DDP, it is also seen that the optimal values of $\lambda$ are specific and the system seems to be quite sensitive. A $\lambda_{o p t}$ from one driving cycle is not necessarily charge sustaining on another, see Fig. 6. The controller has to adapt to the driving scenario.

\subsection{Static Prediction Based on SOC}

In Ambühl et al. (2009) it is suggested that $\lambda_{\text {opt }}$ is approximated by an affine function in SOC. Since the eTVD mainly is an AWD concept, the charge sustenance of the controller is crucial. Therefore another adaptation 


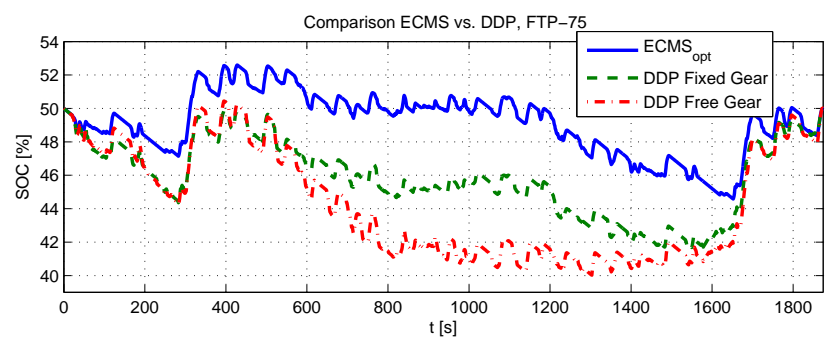

Fig. 5. The optimal SOC trajectory on the FTP-75 cycle with ECMS and DDP. The offline optimization results in a different SOC trajectory than DDP, mainly due to the nominal battery parameters used.

Table 2. $\lambda_{\text {opt }}$ and associated consumption of the different tables. Consumptions are in $\mathrm{L} / 100 \mathrm{~km}$ and Reduction is compared to AWD.

\begin{tabular}{|c|c|c|c|c|}
\hline Cycle & Performance & EMCS $_{\text {opt }}$ & $\mathcal{T}_{\mathbf{L}}$ & $\mathcal{T}_{\mathbf{S}}$ \\
\hline \multirow{3}{*}{ NEDC } & $\lambda_{\text {opt }}$ & 2.8384 & 2.8238 & 2.8426 \\
\cline { 2 - 5 } & Consumption & 5.728 & 5.733 & 5.738 \\
\cline { 2 - 5 } & Reduction & $17.23 \%$ & $17.15 \%$ & $17.08 \%$ \\
\hline \multirow{3}{*}{ FTP-75 } & $\lambda_{\text {opt }}$ & 2.6353 & 2.6615 & 2.7146 \\
\cline { 2 - 5 } & Consumption & 5.505 & 5.512 & 5.534 \\
\cline { 2 - 5 } & Reduction & $24.33 \%$ & $24.23 \%$ & $23.93 \%$ \\
\hline
\end{tabular}
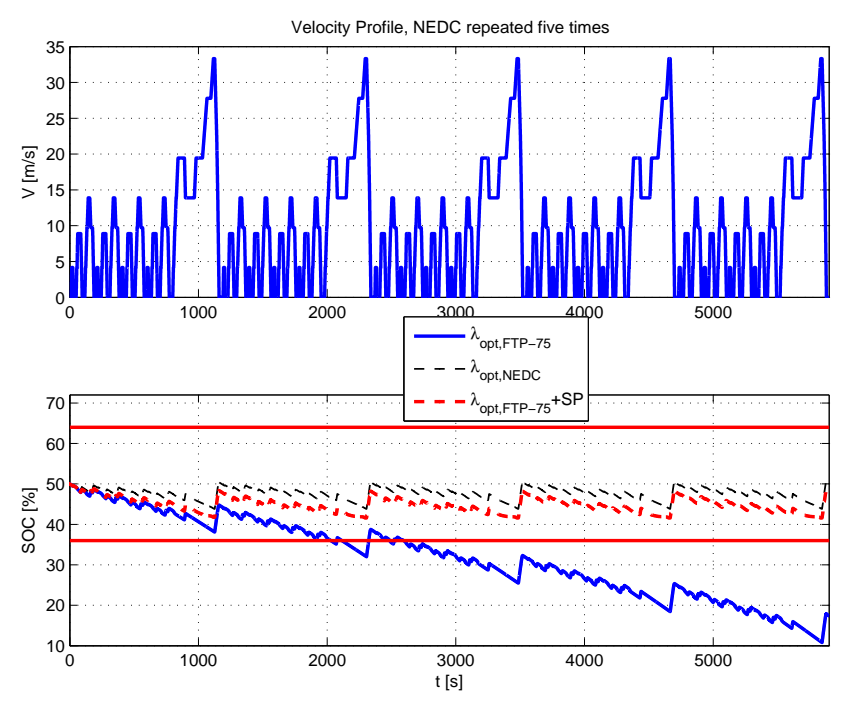

Fig. 6. The NEDC cycle repeated five times with $\lambda_{o p t, N E D C}, \lambda_{o p t, F T P-75}$ and $\lambda_{o p t, F T P-75}$ with the use of Static Prediction based on SOC. The system is sensitive to the equivalence factors. The $\lambda_{o p t}$ for one driving cycle leads to poor performance on another, but with the adaptive control the system is charge sustaining.

function is suggested. Under the assumption that there exists one $\lambda$ that approximates a given driving cycle, the controller should ideally find that $\lambda$ for the future driving mission and use that value for the entire mission. To allow the system to use as much of the battery capacity as possible the idea is to create a function that is relatively flat around the center of the desired SOC window. However, when the SOC approaches the limits of the SOC window it needs to adapt to ensure charge sustenance. The chosen function that fulfills these requirements is a tangens function, see Fig. 7-left. The
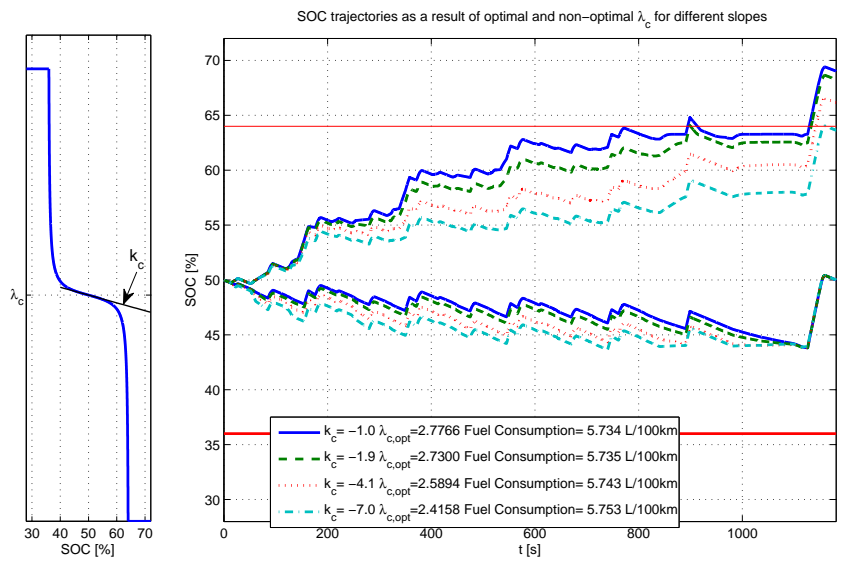

Fig. 7. Left: The shape of the function used for adaptive control. Right: SOC trajectories for different slopes on the NEDC cycle with $\lambda_{c, o p t}$ as well as with $\lambda_{c}=4$. The plot indicates that a steeper slope is more able to keep the SOC within the limits but it also shows that a steep slope alone does not guarantee that the SOC stays within the desired SOC window. A steep slope also increases the consumption.

Table 3. $\lambda_{c, o p t}$ and associated consumptions. Consumptions are in $\mathrm{L} / 100 \mathrm{~km}$ and reduction is compared to AWD.

\begin{tabular}{|c|c|c|c|}
\hline Cycle & Performance & $\mathcal{T}_{\mathbf{L}}$ & $\mathcal{T}_{\mathbf{S}}$ \\
\hline \multirow{3}{*}{ NEDC } & $\lambda_{\mathbf{c}, \text { opt }}$ & 2.73 & 2.7374 \\
\cline { 2 - 4 } & Consumption & 5.735 & 5.739 \\
\cline { 2 - 4 } & Reduction & $17.12 \%$ & $17.07 \%$ \\
\hline \multirow{3}{*}{ FTP-75 } & $\lambda_{\mathbf{c}, \text { opt }}$ & 2.6365 & 2.6823 \\
\cline { 2 - 4 } & Consumption & 5.515 & 5.533 \\
\cline { 2 - 4 } & Reduction & $24.19 \%$ & $23.95 \%$ \\
\hline
\end{tabular}

adaptation of $\lambda$ is of the form:

$$
\lambda=f_{S P}\left(\lambda_{c}, k_{c}, S O C\right)
$$

where $f_{S P}$ has the shape of a tangens function centered at $\lambda_{c}$, with the slope $k_{c}$. As seen in Fig. 7-right the fuel consumption increases with the slope but it is also apparent that the ability of the system to keep the SOC within the desired SOC window, increases with the slope, since a change in SOC results in a larger change in $\lambda$. However, since there is no way of knowing the optimal $\lambda$ for the current driving mission there is no slope that guarantees charge sustenance. The choice is a trade-off between charge sustenance and fuel consumption. Here $k_{c}=-1.9$ is chosen. In Fig. 6 the same test as in Section 6 is shown, now with the use of the Static Prediction based on SOC (SP) in (5). With the use of the new adaptation the system is not as sensitive to the initial $\lambda$. The system is now charge sustaining with $\lambda_{c}=\lambda_{o p t, F T P-75}$. However the fuel consumption increases slightly, see Table 3 compared to Table 2. Also interesting to note is that $\lambda_{c, o p t}$, the $\lambda_{c}$ that gives $\triangle S O C=0$, is not the same as the $\lambda_{o p t}$ that approximates the driving cycle.

\subsection{Adaptive Prediction Based on SOC}

The proposed strategy has introduced some adaptivity to the system, but since there is no way of knowing the $\lambda_{c}$ that approximates the future driving mission, it is not necessarily enough. The value of $\lambda_{c}$ is still important. As 

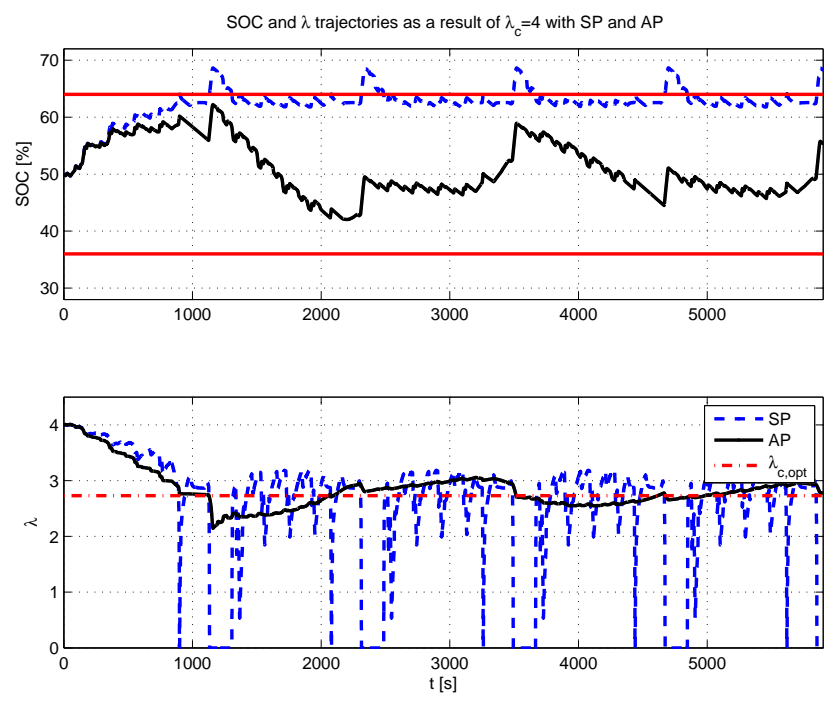

Fig. 8. SOC and $\lambda$ trajectories as a result of $\lambda_{c}=4$, NEDC repeated 5 times. With static prediction based on SOC the system finds a point on the tangens function, around which it varies, that is not necessarily within the desired SOC window. With adaptive prediction based on SOC the control maintains the SOC within the desired SOC window.

seen in Fig. 8 the SOC does not stay within the SOC window when the $\lambda_{c}$ value differs too much from $\lambda_{c, \text { opt }}$. The system instead varies around a SOC value that is not necessarily within the SOC window. The corresponding $\lambda$ value seems to vary around a value close to the $\lambda_{c, o p t}$ found in Section 6.1. The idea is thus to let the center of the function proposed in Section 6.1 change according to the trend of the $\lambda$ values. To find the trend a low-pass filter is used according to:

$$
\begin{aligned}
& \lambda_{c}^{p+1}=(1-\alpha) \lambda_{c}^{p}+\alpha \lambda^{p} \\
& \lambda^{p+1}=f_{S P}\left(\lambda_{c}^{p+1}, k_{c}, S O C\right)
\end{aligned}
$$

The trade-off is between response time and fuel consumption. If the time constant is small, the system will find the optimal $\lambda$ region fast, but a fast filter also means that $\lambda_{c}$ becomes sensitive to the current $\lambda$ which increases the fuel consumption. Here $\alpha$ is chosen so the time constant of the filter is around 200s. To avoid build-up in the lowpass filter, similar to integral wind-up, $\lambda_{c}$ is only allowed to move in what can be considered a feasible region, chosen to be between 2 and 6 . With the use of the Adaptive Prediction based on SOC (AP) in (6)-(7) the control manages to maintain the SOC within the desired SOC window despite the use of a too high initial $\lambda_{c}$, as seen in Fig. 8.

\section{TESTS}

So far the systems have been designed and evaluated using known driving cycles. To investigate how well the systems perform in a more realistic situation the system is tested both on unknown driving cycles as well as in a real vehicle.

\subsection{Randomized Driving Cycle}

To simulate real driving a driving cycle is constructed out of a random set of driving cycles. The 30 selected driving cycles represent roughly 8 hours of driving and
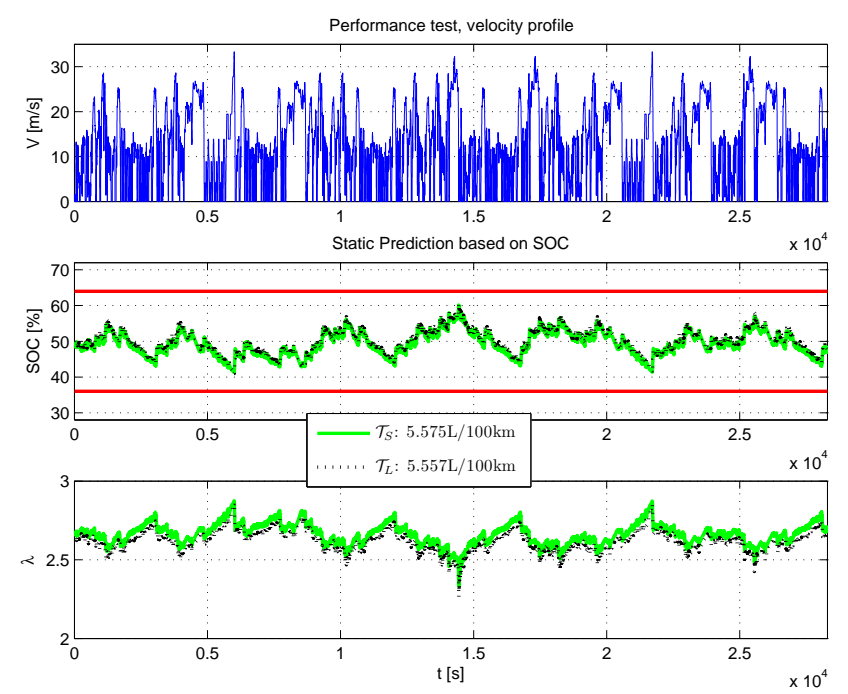

(a)
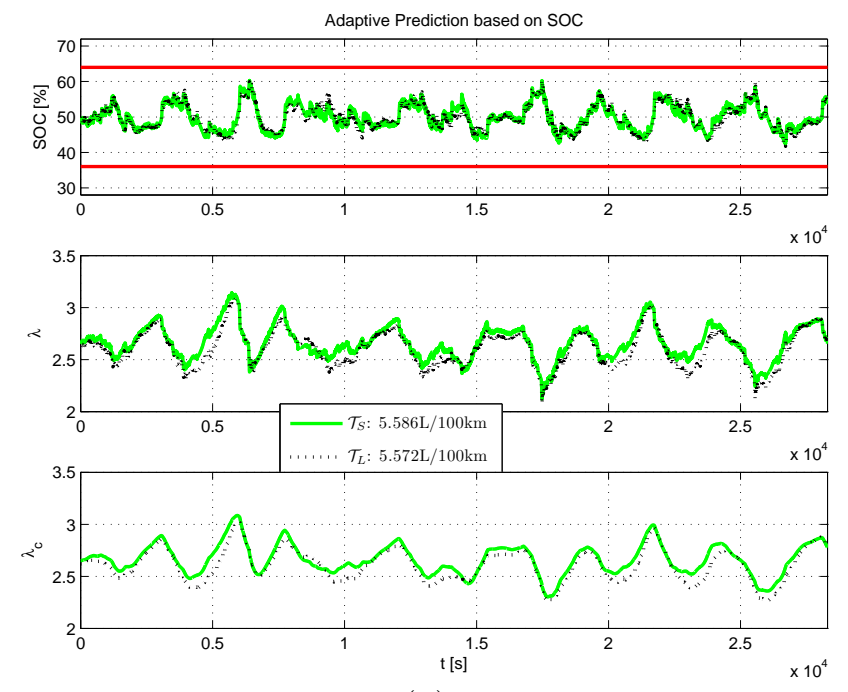

(b)

Fig. 9. Performance test with (a) SP and (b) AP. Both systems are charge sustaining over the randomized driving mission and the $\mathcal{T}_{S}$ result in a slightly higher consumption than the $\mathcal{T}_{L}$.

a distance of $350 \mathrm{~km}$. On this driving mission the ECMS with both adaptive and static prediction based on SOC is tested with both $\mathcal{T}_{L}$ and $\mathcal{T}_{S}$ and the result is shown in Fig. 9 and Table 4. Because of the length of the driving mission the fuel equivalent of the deviation in end SOC is deemed negligible. Both the functions proposed for adaptive control are charge sustaining and imply a fuel consumption reduction of $19-20 \%$ compared to AWD. AP results in a slightly higher consumption than SP, as well as $\mathcal{T}_{S}$ results in a slightly higher consumption than $\mathcal{T}_{L}$.

\subsection{Vehicle Tests}

The system that is chosen to be implemented in the test vehicle is the one with $\mathcal{T}_{S}$ and adaptive prediction based on SOC. The set $\mathcal{T}_{S}$ is used because of the substantial decrease in memory usage and only slight increase in fuel consumption. Even though it is implied in Section 7.1 that static prediction is charge sustaining under normal driving circumstances the extra robustness of the adaptive 
Table 4. The consumptions for $\mathcal{T}_{L}$ and $\mathcal{T}_{S}$ with the two different adaptive controllers during a set of randomized driving cycles. Red. is the reduction compared to AWD

\begin{tabular}{|c|c|c|c|}
\hline Config. & Tables & Consumption & Red. \\
\hline AWD & - & $6.963 \mathrm{~L} / 100 \mathrm{~km}$ & - \\
\hline \multirow{2}{*}{ ECMS w. SP } & $\mathcal{T}_{\mathbf{S}}$ & $5.575 \mathrm{~L} / 100 \mathrm{~km}$ & $19.19 \%$ \\
\cline { 2 - 4 } & $\mathcal{T}_{\mathbf{L}}$ & $5.557 \mathrm{~L} / 100 \mathrm{~km}$ & $20.19 \%$ \\
\hline \multirow{2}{*}{ ECMS w. AP } & $\mathcal{T}_{\mathbf{S}}$ & $5.586 \mathrm{~L} / 100 \mathrm{~km}$ & $19.78 \%$ \\
\cline { 2 - 4 } & $\mathcal{T}_{\mathbf{L}}$ & $5.572 \mathrm{~L} / 100 \mathrm{~km}$ & $19.98 \%$ \\
\hline
\end{tabular}
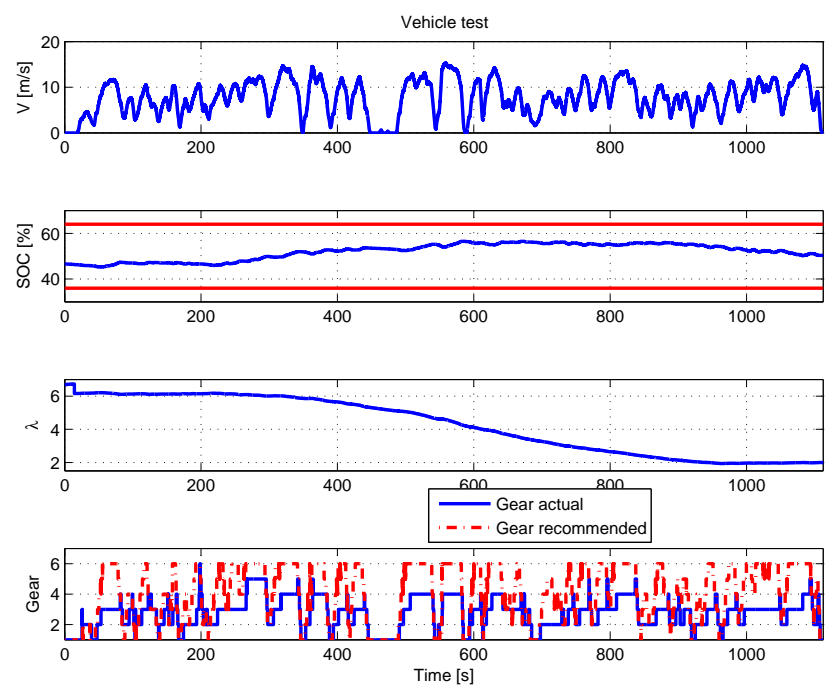

Fig. 10. The SOC and control trajectories during the vehicle test. The system is charge sustaining despite a high $\lambda_{c}$ but the gear recommendation is often too high for comfort.

prediction is considered desirable. The test drive, see Fig. 10, represents urban driving with many transients and low speed, and is done to test the driveability and the charge sustenance of the control system. The test drive is done with an initial SOC at reference level but a high $\lambda_{c}$. As seen in the figure the control is charge sustaining despite the initially high $\lambda_{c}$. The AP adapts $\lambda$ and maintains the SOC within the desired SOC window. It is also seen that the gear recommendation often is too high for comfort. For more test data see Sivertsson (2010).

\section{CONCLUSION}

A map-based implementation of ECMS is developed and the effects of the discretization are studied. Performing the optimization offline with stationary operating points and average battery parameters increases the consumption by only a few percent compared to DDP if the same gear trajectory is used. DDP implies a potential to decrease the consumption further by a couple of percent if no restrictions on gear selection is enforced, but the resulting gear trajectory is highly unrealistic, making the solution infeasible.

The effect on the consumption by reducing the size of the tables is small. Making the discretization sparser in the $\lambda$-dimension according to the strategy proposed in Section 4.2 only increases the consumption by $0.1 \%$ and making all three dimensions sparser only results in an increase of less than $1 \%$.

Both methods suggested for adaptive control are charge sustaining and only result in a slight increase in consumption compared to when $\lambda_{o p t}$ is used. The static prediction based on SOC increases the consumption less than the adaptive prediction based on SOC, but the latter is more robust. The use of static prediction based on SOC reduces the importance of knowing the optimal equivalence factor for the future driving mission, as the control manages to maintain the SOC within the desired window as long as the used $\lambda_{c}$ doesn't differ too much from the $\lambda_{c, o p t}$ of the driving mission. With the use of adaptive prediction based on SOC the need for information about the future mission is eliminated. Instead the control adapts so that $\lambda$ varies around $\lambda_{o p t}$ of the driving mission, ensuring charge sustenance.

\section{ACKNOWLEDGEMENTS}

The help and support from Haldex Traction AB is gratefully acknowledged.

\section{REFERENCES}

Ambühl, D., Sciarretta, A., Onder, C., Guzzella, L., Sterzing, S., Mann, K., Kraft, D., and Küsell, M. (2009). A causal operation strategy for hybrid electric vehicles based on optimal control theory. In IFAC Workshop on Engine and Powertrain Control, Simulation and Modeling, 109-117.

Chasse, A., Hafidi, G., Pognant-Gros, P., and Sciarretta, A. (2009). Supervisory control of hybrid powertrains: an experimental benchmark of offline optimization and online energy management. In IFAC Workshop on Engine and Powertrain Control, Simulation and Modeling, 109-117.

Guzzella, L. and Sciarretta, A. (2007). Vehicle Propulsion Systems - Introduction to Modeling and Optimization. Springer-Verlag, 2nd edition.

Musardo, C. and Rizzoni, G. (2005). A-ECMS: An adaptive algorithm for hybrid electric vehicle energy management. In IEEE Conference on Decision and Control and the European Control Conference, 44, 18161823.

Paganelli, G., Delprat, S., Guerra, T., Rimaux, J., and Santin, J. (2002). Equivalent consumption minimization strategy for parallel hybrid powertrains. In IEEE Conference on Vehicular Technology, 55, 2076-2081.

Paganelli, G., Tateno, M., Brahma, A., Rizzoni, G., and Guezennec, Y. (2001). Control development for a hybrid-electric sport-utility vehicle: Strategy, implementation and field test results. In Proceedings of the American Control Conference, 5064-5069.

Pisu, P. and Rizzoni, G. (2007). A comparative study of supervisory control strategies for hybrid electric vehicles. IEEE Transactions on Control Systems Technology, 15(3), 506-518.

Sciarretta, A., Back, M., and Guzzella, L. (2004). Optimal control of parallel hybrid electric vehicles. IEEE Transactions on Control Systems Technology, 12(3), 352-363.

Sivertsson, M. (2010). Optimization of Fuel Consumption in a Hybrid Powertrain. Master's thesis, Linköpings Universitet, SE-581 83 Linköping. 